\title{
PTEE Material Life Modeling based on Compound Poisson Process of Fatigue Crack
}

\author{
Longbo Liu 1, a, Shuizhuang Miao 2, b \\ ${ }^{1}$ Institute of Naval Vessels, Naval Academy of Armament, Beijing 100191, China \\ ${ }^{2}$ School of Reliability and Systems Engineering, Beihang University, Beijing 100191, China \\ amsz101371@163.com, b343563688@qq.com
}

\begin{abstract}
Keywords: Compound Poisson Process, Reliability Modeling, Cumulative Damage, Fatigue Crack.
\end{abstract}

\begin{abstract}
In order to study the life modeling problem of the PTEE material, this paper proposes a Compound Poisson process life modeling method for PTEE material, which performance degradation process is modeled by the PTEE material impact fatigue crack. In the impact work condition, the number of impact times of the material obeys the Poisson process and the damage caused by each impact has a cumulative effect. By using fatigue cumulative damage theory, we can obtain the Compound Poisson process model. Then, the parameters point estimation of Compound Poisson process model are evaluated by using moment estimation method combined with least- square principle method and we utilize fatigue crack data of FTEE material to evaluate the life of PTEE material. A case study on PTEE sealing material for a ship gas turbine generator with reliability degradation testing is used to demonstrate the accuracy and effectiveness of the proposed method. This method solves the problem of material life modeling and evaluation of fatigue crack due to impact characteristics. It can be used as a reference for the reliability evaluation of the similar material products.
\end{abstract}

\section{Introduction}

Polytetrafluoroethylene (PTEE) is an important species of polymers. It has excellent properties of high temperature, chemical stability and durability. Its unique properties make it a major filler material for aerospace, marine and other sealed package. PTEE mechanical properties will gradually decline and cause fatigue crack affected by the impact of external mechanical environmental factors during operation. Therefore, the fatigue cracks mostly affect the life of the PTEE material. At present, life assessment methods of PTEE material are mainly chemical performance test and aging test through considering the material itself, lacking the consideration of the overall mechanical environment for materials used in products.

In the aspect of material life modelling based on fatigue cracks, some scholars have conducted a series of studies. S Boljanović. et al proposed a computational procedures for the strength analysis of two cracked lug configurations [1]. K Kirane. et al composed a set of test data to verify the effect on fatigue lifetimes and composed a reliable evaluation of zone size in cyclic fracture process [2]. M Sistaninia. et al developed a new model based on element-based mesoscale to simulate the deformation in some localizations of material and the growth of microstructurally fatigue crack by considering the anisotropic behavior of the individual grains [3]. J B Jordon. et al employed a microstructure-based fatigue model to evaluate the fatigue cracks damage in 4140 steel [4]. J A R Duran. et al composed some useful suggestions for applying the three current available methods into reducing the second dependence through the adoption of tuning constants [5]. J Zhang. et al composed a comparatively method to investigate the relationships between the recovery properties of asphalt binder elastic and the fatigue performance of HMA [6].

In this paper, the fatigue crack damage of PTFE material caused by the impact in the process of equipment operation is presented, and a method of fatigue crack life modeling based on the Compound Poisson process is proposed. In the impact work condition, the number of impact times of the material obeys the Poisson process and the damage caused by each impact has a cumulative effect. By combing 
the Poisson process and cumulative process, we obtained the Compound Poisson process model. By using moment estimation method combined with least- square principle method, we got the parameters point estimation of the Compound Poisson process model. This method solves the problem of PTEE material life modeling and evaluation of fatigue cracks due to the impact characteristics, and has a reference value for the life evaluation of materials with similar characteristics.

\section{Reliability modeling of materials fatigue crack}

\subsection{Compound Poisson process.}

In engineering practice, a lot of equipment materials are often in their work environment to withstand continuous impact damage. It can be considered that the impact of the material on the fatigue crack damage in the work environment follows the Poisson process $\{N(t), t \geq 0\} . N(t)$ is the number of impact times during time $[0, t]$. The material damage degree is different during each impact time, and the damage degree is a random variable. It can be assumed that the degree of damage caused by the impact of the material at each time is a random variable $\left\{S_{i}, i=1,2, \cdots\right\}$. In addition, according to the mechanism of impact source in engineering practice, it can be considered that the degree of fatigue crack damage caused by impact is independent and identically distributed. That is to say, the material damage degree $S_{k}$ is independent to impact time $N(t)$. Obviously, in the engineering practice, the damage degree of equipment materials caused by the continuous impact is a kind of cumulative effect. Therefore, the damage of the material at time $t$ should be the result of the $S_{1}, S_{2}, \cdots, S_{N(t)}$ overlay caused by $N(t)$ impacts. This is:

$$
X(t)=\sum_{i=1}^{N(t)} S_{i}
$$

Random variable $X(t)$ is a Compound Poisson process which is composed of random processes $\{N(t), t \geq 0\}$ and $\left\{S_{i}, i=1,2, \cdots\right\}$.

$D_{f}$ represents the failure threshold, then the probability function $R(t)$ of Compound Poisson process can be expressed as:

$$
\begin{aligned}
R(t) & =P\left(X(t) \leq D_{f}\right)=P\left(\sum_{i=1}^{N(t)} S_{i} \leq D_{f}\right) \\
& =\sum_{k=1}^{\infty} P\left(\sum_{i=1}^{N(t)} S_{i} \leq D_{f}, N(t)=k\right) \\
& =\sum_{k=1}^{\infty} P\left(\sum_{i=1}^{N(t)} S_{i} \leq D_{f} \mid N(t)=k\right) P(N(t)=k) \\
& =\sum_{k=1}^{\infty} \Phi\left(\frac{D_{f}-k \mu}{k \sigma}\right) \frac{(\lambda t)^{k}}{k !} e^{-\lambda t}
\end{aligned}
$$

Where $N(t)$ is the impact time and obeys Poisson process, $S_{i}$ is damage degree of each time and obeys normal distribution, $P$ is the probability function, $\lambda$ is the parameter of Poisson process and $\mu, \sigma$ is the parameters of normal distribution.

\subsection{Statistical analysis method of model parameters.}

In order to obtain the reliability characteristics of PTFE material which is in accordance with the above mentioned damage characteristics, the unknown parameters $\lambda, \mu, \sigma$ in the formula (2) should be estimated with the test data.

According to the relationship between the characteristic function of the random variables and each order moment, overall moments of the Compound Poisson process is obtained. By measuring the PTEE material fatigue crack data, we can get the parameters point estimation of Compound Poisson process model by using moment estimation method combined with least- square principle method and use fatigue crack data to evaluate the life of PTEE material. 
Assuming that the initial distribution of material performance degradation is $N\left(\alpha, \sigma_{\alpha}^{2}\right)$, where $\alpha$ is mean value and $\sigma_{\alpha}^{2}$ is variance. The normal distribution is the characterization of the initial performance of the product and is related to processing technology, production level and other factors. According to the definition of the Compound Poisson process, each random variable $S_{k}$ has the same distribution density. If $N(t)=k$, then $X(t)$ is the sum of the $k$ random variables. The characteristic function $\phi_{x}(v, t)$ of $N(t)$ can be expressed as:

$$
\begin{aligned}
\phi_{x}(v, t) & =\sum_{k=0}^{\infty} E\left[e^{j v X(t)} \mid N(t)=k\right] P[N(t)=k] \\
& =\sum_{k=0}^{\infty} E\left[e^{j v \sum_{i=1}^{k} S_{i}} \mid N(t)=k\right] P[N(t)=k] \\
& =\sum_{0}^{\infty}\left\{\prod_{i=1}^{k} E\left[e^{j v S_{i}}\right] P[N(t)=k]\right\} \\
& =\sum_{k=0}^{\infty}\left[\phi_{s}(v)\right]^{k} \frac{(\lambda t)^{k}}{k !} e^{-\lambda t} \\
& =e^{-\lambda t} \sum_{k=0}^{\infty} \frac{\left(\lambda t \phi_{s}(v)\right)^{k}}{k !} \\
& =e^{-\lambda t} \cdot e^{\lambda t \phi_{s}(v)}=e^{\lambda t\left[\phi_{s}(v)-1\right]}
\end{aligned}
$$

Derivative the characteristic function $\phi_{x}(v, t)$, we can get the first three moments. The first three order moments of $X(t)$ is:

$$
\begin{aligned}
E[X(t)]= & \left.(-j) \frac{\partial}{\partial v} \phi_{x}(v, t)\right|_{v=0} \\
= & \left.(-j)\left\{\lambda t \frac{\partial \phi_{S}(v)}{\partial v} e^{\lambda t\left[\phi_{s}(v)-1\right]}\right\}\right|_{v=0}=\lambda t E[S] \\
E\left[X^{2}(t)\right]= & \left.(-j) \frac{\partial^{2}}{\partial v^{2}} \phi_{x}(v, t)\right|_{v=0} \\
= & (\lambda t)^{2}[E(S)]^{2}+\lambda t E\left[S^{2}\right] \\
E\left[X^{3}(t)\right]= & \left.(-j) \frac{\partial^{3}}{\partial v^{3}} \phi_{x}(v, t)\right|_{v=0} \\
= & (\lambda t)^{3}[E(S)]^{3}+\left(\lambda t+\lambda^{2} t^{2}\right) E[S] E\left[S^{2}\right] \\
& +\lambda T E\left[S^{3}\right]
\end{aligned}
$$

The random variable $S$ is with respect to the normal distribution $S \sim N\left(\mu, \sigma^{2}\right)$, then:

$$
E[S]=\mu, E\left[S^{2}\right]=\mu^{2}+\sigma^{2}, E\left[S^{3}\right]=\mu^{3}+2 \mu \sigma^{2}
$$

Thus:

$$
\begin{aligned}
E\left[X^{3}(t)\right] & =\lambda^{3} \mu^{3} t^{3}+\left(3 \lambda^{2} t^{2}\right) \mu\left(\mu^{2}+\sigma^{2}\right)=\lambda t\left(\mu^{3}+3 \mu \sigma^{2}\right) \\
& =t\left(\lambda \mu^{3}+3 \lambda \mu \sigma^{2}\right)+t^{2}\left(3 \lambda^{2} \mu^{3}+3 \lambda^{2} \mu \sigma^{2}\right)+t^{3} \lambda^{3} \mu^{3}
\end{aligned}
$$

For any $t_{j}$, the three order sample moment $\hat{E}\left[X^{3}\left(t_{j}\right)\right]=s_{i j}^{3} / n$ can be obtained by the measured value $\left\{s_{i j}, i=1,2, \cdots, n\right\}$. According to the Data columns $\left\{\left(\frac{\hat{E} X^{3}\left(t_{j}\right)}{t_{i}}, t_{j}\right), j=1,2, \cdots, m\right\}$ and the second-order polynomial method, we can obtain:

$$
\frac{\hat{E}\left[X^{3}(t)\right]}{t}=k_{1}+k_{2} t+k_{3} t^{2}
$$


Where, $k_{1}, k_{2}, k_{3}$ are polynomial coefficients obtained by least square method. Based on the principle of moment estimation, we can obtain the following nonlinear equations:

$$
\left\{\begin{array}{c}
\lambda \mu^{3}+3 \lambda \mu \sigma^{2}=k_{1} \\
3 \lambda^{2} \mu^{3}+3 \lambda^{2} \mu \sigma^{2}=k_{2} \\
\lambda^{3} \mu^{3}=k_{3}
\end{array}\right.
$$

Estimated value $\hat{\lambda}, \hat{\mu}, \hat{\sigma}$ of $\lambda, \mu, \sigma$ can be obtained by the solution of the equation group.

\section{Test analysis of the PTEE sealing material for ship gas turbine generator}

The PTEE sealing material for a ship gas turbine generator withstands sustained cold and heat impact damage. According to the mechanism of impact damage caused by material damage, it can be considered that the impact of the PTEE sealing material on the fatigue crack damage in the work environment follows the Poisson process $\{N(t), t \geq 0\}$. The degree of damage caused by the impact of the PTEE sealing material at each time is a random variable $\left\{S_{i}, i=1,2, \cdots\right\}$. Thus, the fatigue crack growth process occurs at the contact position under thermal fatigue and thermal impact. PTFE sealing materials of the gas turbine generator obeys the Compound Poisson process. $D_{f}$ represents the failure threshold, then the probability function $R(t)$ of Compound Poisson process can be expressed as equation (2).

The gas turbine generator used in warship cost so much and thus only limited test can be conducted to obtain the fatigue crack data by only one sample. The whole test time is $1000 \mathrm{~h}$. Each stage time is $t_{1}=100 h, t_{2}=200 h, t_{3}=300 h, \ldots, t_{10}=1000 h$. In each stage, the fatigue crack of the impact part of the PTFE sealing material of gas generator is measured. Fatigue crack data obtained is shown in Table 1.

Table 1. The fatigue crack data of PTEE sealing material

\begin{tabular}{|c|c|c|c|c|c|}
\hline Time(h) & 100 & 200 & 300 & 400 & 500 \\
\hline Crack (mm) & 0.015 & 0.018 & 0.021 & 0.023 & 0.025 \\
\hline time(h) & 600 & 700 & 800 & 900 & 1000 \\
\hline Crack (mm) & 0.028 & 0.030 & 0.035 & 0.039 & 0.045 \\
\hline
\end{tabular}

The parameters needed to be estimated in the Compound Poisson process model are $\mu, \sigma$ and $\lambda$. we can get the parameters point estimation of Compound Poisson process model by using moment estimation method combined with least- square principle method. Using the above method, the results of parameters estimation in the Compound Poisson process are shown in Table 2:

Table 2. Results of parameters estimation in the Compound Poisson process

\begin{tabular}{|c|c|c|c|}
\hline Parameter & $\hat{\mu}$ & $\hat{\sigma}$ & $\hat{\lambda}$ \\
\hline Evaluation & 0.0062210 & 0.0000169 & 0.000112 \\
\hline
\end{tabular}

According to the design requirements of the gas turbine generator, the failure threshold $D_{f}$ of the PTEE sealing material is $D_{f}=0.08 \mathrm{~mm}$. Substitute the results of parameter estimation into the PTEE material Compound Poisson process, the reliability function can be obtained:

$$
R(t)=\sum_{k=1}^{\infty} \phi\left(\frac{D_{f}-k \mu}{k \sigma}\right) \frac{(\lambda t)^{k}}{k !} e^{-\lambda t}
$$

The reliability function curve is obtained as shown in Figure 1: 


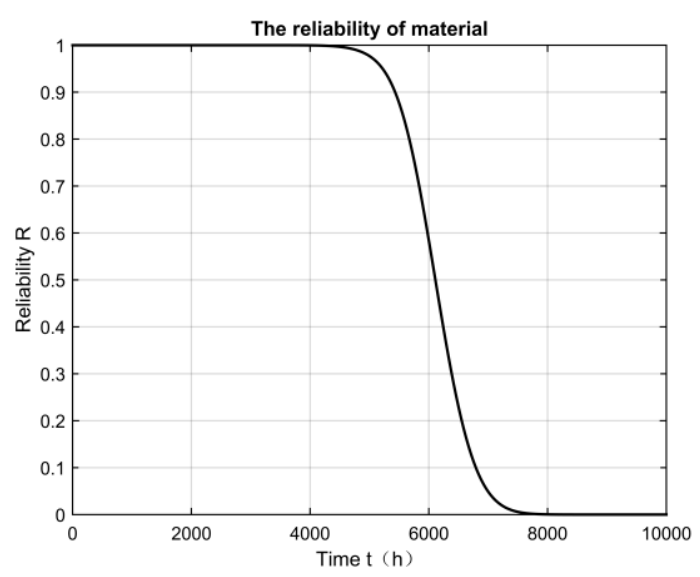

Fig. 1 The reliability function curve of PTEE sealing material

\section{Conclusion}

This paper proposes a Compound Poisson process life modeling method of the PTEE material fatigue crack. In the impact work condition, the number of impact times of the material obeys the Poisson process and the damage caused by each impact has a cumulative effect. By combing this two processes, the Compound Poisson process model is obtained. By using moment estimation method combined with least- square principle method, we get the parameters point estimation of the Compound Poisson process model and utilize fatigue crack data to evaluate the life of PTEE material.

The accuracy and effectiveness of the method are verified by an example of a PTEE sealing material for ship gas turbine generator. The fatigue crack data of the sealing material is measured and the life modeling of the gas turbine generator is carried out by using the method above. This method solves the problem of material life modeling and evaluation of fatigue crack due to impact characteristics. It can be used as a reference for the reliability evaluation of the similar material products.

\section{References}

[1]. Boljanović S, Maksimović S. Fatigue crack growth modeling of attachment lugs [J]. International Journal of Fatigue. Vol. 58(2014), p. 66-74.

[2]. Kirane K, Bažant Z P. Size effect in Paris law and fatigue lifetimes for quasibrittle materials: Modified theory, experiments and micro-modeling [J]. International Journal of Fatigue. Vol. 83(2016), p. 209-220.

[3]. Sistaninia M, Niffenegger M. Prediction of damage-growth based fatigue life of polycrystalline materials using a microstructural modeling approach [J]. International Journal of Fatigue. Vol. 66(2014) No. 6, pp. 118-126.

[4]. Jordon J B, Horstemeyer M F. Microstructure-Sensitive Fatigue Modeling of AISI 4140 Steel [J]. Journal of Engineering Materials \& Technology. Vol. 136(2014) No. 2, pp. 92-94.

[5]. Duran J A R, Hernandez C T. Evaluation of three current methods for including the mean stress effect in fatigue crack growth rate prediction [J]. Fatigue \& Fracture of Engineering Materials \& Structures. Vol. 38(2015) No. 4, pp. 410-419.

[6]. Zhang J, Simate G S, Sang I L, et al. Relating asphalt binder elastic recovery properties to HMA crack modeling and fatigue life prediction[J]. Construction \& Building Materials. Vol. 111(2014), p. 644-651. 